\title{
Macrophages Promote Growth of Squamous Cancer Independent of T cells
}

\author{
F.L. Wu ${ }^{1,2}$, K. Nolan ${ }^{2}$, A.A. Strait ${ }^{2}$, L. Bian ${ }^{2}$, K.A. Nguyen ${ }^{2}$, J.H. Wang ${ }^{3}$, A. Jimeno ${ }^{4}$, \\ H.M. Zhou', C.D. Young ${ }^{2}$, and X.J. Wang ${ }^{2,5}$
}

\begin{abstract}
Oral cancers, primarily squamous cell carcinomas (SCCs), progress either slowly or aggressively. Here we assessed the role of macrophages in SCC behavior. We used mouse SCC cells derived from tumors harboring a Kras ${ }^{\text {Gi2D }}$ activation mutation and Smad4 deletion in keratin 15-positive stem cells and a human oral SCC cell line, FaDu, which has NRAS amplification and SMAD4 deletion. SCC cells were transplanted into immune-compromised or immune-competent (syngeneic) recipients. After tumors were established, we used clodronate liposomes to ablate macrophages. We found that the number of tumor-associated macrophages (TAMs) was not affected by the presence of $\mathrm{T}$ cells but differed considerably among tumors derived from different SCC lines. Clodronate significantly reduced TAMs and splenic macrophages, resulting in reduced SCC volumes. Tumors with clodronate treatment did not show decreased proliferation but did exhibit increased apoptosis and reduced vascular density. FLIP (Fas-associated via death domain-like interleukin I $\beta$ converting enzyme inhibitory protein), an apoptosis inhibitor abundantly produced in tumor cells and TAMs, was reduced in tumor cells

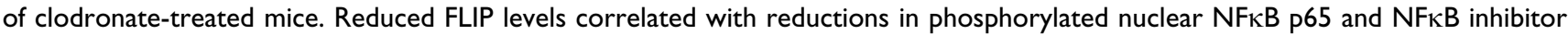
attenuated FLIP protein levels in SCC cells. Furthermore, TGF $\beta$ I serum levels and PSmad3 were reduced in clodronate-treated mice, but their reductions were insufficient to reverse epithelial-mesenchymal transition or TGF $\beta$-mediated angiogenesis in endothelial cells. Consequently, metastasis was not significantly reduced by macrophage reduction. However, reduced pSmad3 correlated with reduction of its transcriptional target, vascular endothelial growth factor A, in clodronate-treated tumor cells, which correlated with reduced vascular density in clodronate-treated tumors. Taken together, our study revealed that macrophages contribute to SCC expansion through interactions with tumor cells but are dispensable for SCC metastasis. Our study provides novel insights into understanding the contributions and limitations of TAMs in SCC progression.
\end{abstract}

Keywords: immunity, metastasis, apoptosis, angiogenesis, squamous cell carcinoma, clodronic acid

\section{Introduction}

Squamous cell carcinomas (SCCs) arise from stratified epithelia of the skin, oral cavity, airways, and cervix. Clinically, SCCs can be indolent - that is, slow growth and no metastasis or aggressive with rapid growth and/or metastasis. We previously generated an aggressive SCC mouse model driven by $\operatorname{Kras}^{\mathrm{G} 12 \mathrm{D}}$ mutation and Smad4 deletion $\left(\mathrm{Smad} 4^{-/-}\right)$in keratin 15 (K15)-positive stem cells of the skin or oral cavity (White et al. 2013). SMAD4 genetic deletion occurs in $30 \%$ to $50 \%$ of tobacco-related head and neck SCCs (Hernandez et al. 2018). $R A S$ mutations in oral SCCs are low in Western countries but occur in $>50 \%$ of oral cancer cases in south Asian populations (Saranath et al. 1991). Targeting Kras ${ }^{\mathrm{G} 12 \mathrm{D}}$ and $\mathrm{Smad} 4^{-/-}$to $\mathrm{K} 15^{+}$ stem cells that reside in the hair follicle bulge (Jih et al. 1999) or the deeper part of the rete in tongue papillae (Tudor et al. 2004) caused aggressive SCCs that are dedifferentiated and metastasize to the lung (White et al. 2013). Because these SCCs develop in an immune-competent background, it suggests that they evade antitumor immunity, and $\mathrm{T}$ cells do not appear to restrict tumor progression in this model. These SCCs contain numerous tumor-associated macrophages (TAMs) that led us to assess if TAMs contribute to the aggressive behaviors of these tumors.
'State Key Laboratory of Oral Diseases, Department of Oral Medicine, West China Hospital of Stomatology, Sichuan University, Chengdu, China

${ }^{2}$ Department of Pathology, University of Colorado Anschutz Medical Campus, Aurora, CO, USA

${ }^{3}$ Department of Immunology and Microbiology, University of Colorado Anschutz Medical Campus, Aurora, CO, USA

${ }^{4}$ Division of Medical Oncology, Department of Medicine, University of Colorado Anschutz Medical Campus, Aurora, CO, USA

${ }^{5}$ Veterans Affairs Medical Center, VA Eastern Colorado Health Care System, Aurora, CO, USA

A supplemental appendix to this article is available online.

Corresponding Authors:

H.M. Zhou, State Key Laboratory of Oral Diseases, Department of Oral Medicine, West China Hospital of Stomatology, Sichuan University, South Renmin Road, Sec. 3, No. 14, Chengdu, Sichuan 61004I, China.

Email: zhouhm@scu.edu.cn

C.D. Young, Department of Pathology, University of Colorado Anschutz Medical Campus, 12800 E. 19th Ave, RCI-North, P 18-5403, Aurora, CO 80045, USA.

Email: Christian.Young@ucdenver.edu

X.J. Wang, Veterans Affairs Medical Center, VA Eastern Colorado Health Care System, Aurora, CO 80045, USA.

Email: xiao-jing.wang@va.gov 
TAMs polarize into inflammation-promoting M1-type and immunosuppressive M2-type macrophages (Mantovani et al. 2004; Biswas and Mantovani 2010; Chanmee et al. 2014). Polarization is driven by signaling in the tumor microenvironment. For example, interferon gamma and tumor necrosis factor $\alpha(\mathrm{TNF} \alpha)$ drive an M1 phenotype, whereas IL-4, IL-13, and TGF- $\beta$ promote an M2 phenotype (Biswas and Mantovani 2010; Chanmee et al. 2014; Zhang et al. 2014; Yuan et al. 2015; Murray 2017). Because the tumor microenvironment fluctuates, M1 and M2 phenotypes often coexist (Qian and Pollard 2010). Macrophages have been shown to play important roles in cancer initiation and metastasis (Qian and Pollard 2010; Noy and Pollard 2014; Ruffell and Coussens 2015). However, the temporal and spatial roles of TAMs in different cancer types and stages are context specific. For example, although TAMs are associated with progression in oral SCCs (Pirila et al. 2015; Weber et al. 2016), TAMs did not directly promote SCC invasion. TAMs are reported to promote angiogenesis in SCCs (Liss et al. 2001; Okubo et al. 2016). Currently, it is unknown how TAMs affect SCC epithelial cells directly.

To study the roles of TAMs in SCCs, we transplanted SCC cells derived from K15. $\mathrm{Kras}^{\mathrm{G} 12 \mathrm{D}} / \mathrm{Smad}^{-/-} \mathrm{SCC}$ into immunecompetent (C57BL/6J) and immune-compromised (athymic nude) mouse recipients, followed by TAM ablation with clodronate liposomes. We also included a human oral SCC line, $\mathrm{FaDu}$, which has homozygous SMAD4 deletion and NRAS amplification (Barretina et al. 2012). We provide evidence that SCC growth can be attenuated by TAM reduction independent of $T$ cells. Mechanistically, TAM reduction caused increased apoptosis in tumor cells and reduced angiogenesis in tumor stroma but did not affect tumor cell proliferation or metastasis. Our study provides novel insights toward understanding the contributions and limitations of TAMs in SCC progression.

\section{Methods and Materials}

\section{Transplanting SCC Tumor Cells}

All tumor cell lines were cultured in Dulbecco's modified Eagle medium containing 10\% fetal bovine serum. Animal experiments were performed following protocols approved by the Institutional Animal Care and Use Committee of the University of Colorado Denver Anschutz Medical Campus. Mouse SCC cell lines A223 and B931 derived from female mice (White et al. 2013) were injected into female athymic nude or female $\mathrm{C} 57 \mathrm{BL} / 6 \mathrm{~J}$ mice at 8 to $10 \mathrm{wk}$ of age. $\mathrm{FaDu}$, a human oral SCC line, was injected into female athymic nude mice at 8 to $10 \mathrm{wk}$ of age. Two thousand A223 or B931 cells or 1 million FaDu cells were resuspended to a final volume of 40 $\mu \mathrm{L}$ in $50 \%$ matrigel (Corning) $/ 50 \%$ phosphate buffered saline (PBS) and injected subcutaneously into the flanks of mice. Two weeks after tumor cell transplant, we intraperitoneally injected $200 \mu \mathrm{L}$ of clodronate liposomes $(5 \mathrm{mg} / \mathrm{mL})$ or control liposomes (Fisher Scientific) weekly until the end of the experiment. When tumors reached $2.0 \mathrm{~cm}$ in diameter or mice developed metastasis, mice were euthanized to harvest tumors and spleens. Necropsies were performed to detect distant metastasis (lungs and other major organs).

\section{Flow Cytometry and Mass Cytometry (CyTOF)}

Tumor cell suspensions and splenocytes were prepared as described (White et al. 2013). Antibody and cell staining details are described in the Appendix Materials and Methods. The antibody list used for CyTOF is provided in the Appendix Table. viSNE analyses were performed with Cytobank software.

\section{Histology, Immunostaining, TUNEL Assay, ELISA, and Western Blot}

Mouse tumor and tissue samples were dissected and fixed in $10 \%$ neutral buffered formalin overnight. Paraffin-embedded tissues were cut into $6-\mu \mathrm{m}$ sections for hematoxylin and eosin staining or immunostaining procedures as previously described (White et al. 2013) and are detailed in the Appendix Materials and Methods. TUNEL assay kit (terminal deoxynucleotidyl transferase-mediated dUTP-biotin nick end labeling; Promega) was used to detect apoptotic cells according to the manufacturer's instructions. TGF $\beta 1$ levels in the sera of mice was determined by ELISA following the manufacturer's instructions (R\&D Systems).

Small pieces of frozen tumor (100 to $300 \mathrm{~mm}^{3}$ ) were lysed with lysis buffer $\mathrm{M}$ containing phosphatase and protease inhibitor cocktails (Roche Diagnostics) and cleared by centrifugation. Cultured cells were treated with DMSO, $1 \mu$ M LY2109761 (MedChemExpress), 5 $\mathrm{MM}$ BMS345541 (MedChemExpress), 2-ng/mL TGF $\beta$ (R\&D Systems), or 2-ng/mL TNF $\alpha$ (R\&D Systems) for $18 \mathrm{~h}$ before being lysed. Lysates were subjected to SDS-PAGE, transferred to PVDF membranes (Bio-Rad), and probed with antibodies, as detailed in the Appendix Materials and Methods.

\section{Statistical Analysis}

Statistical differences between 2 groups of data were analyzed by a 2-tailed Student's $t$ test or chi-square test. Data are presented as mean \pm SEM. $P$ values $<0.05$ were considered statistically significant.

\section{Results}

\section{Macrophage Reduction Inhibited SCC Tumor Growth but Not Metastasis}

Between 2 mouse SCC lines, A223 cells grow rapidly in C57BL/6J mice (Mishra et al. 2016), whereas B931 cells do not. B931 cells metastasize spontaneously to the lung of immune-compromised recipients (White et al. 2013). We injected these cell lines into the flanks of athymic nude mouse or $\mathrm{C} 57 \mathrm{BL} / 6 \mathrm{~J}$ mouse recipients. When A223 tumors reached $\sim 2,000 \mathrm{~mm}^{3}$, we profiled tumor-infiltrating leukocytes with CyTOF (Fig. 1). CD $45^{+}$leukocytes composed $10 \%$ to $25 \%$ of the total tumor cells, and TAMs, marked by F4/80, were $5 \%$ to $10 \%$ of the $\mathrm{CD} 45^{+}$cells (Fig. 1A). There were typically 2 - to 3-fold more M2 TAMs (CD11b $\left.{ }^{+} \mathrm{F} 4 / 80^{+} \mathrm{Gr}-1^{-} \mathrm{Arg} 1^{+}\right)$than M1 TAMs $\left(\mathrm{CD} 11 \mathrm{~b}^{+} \mathrm{F} 4 / 80^{+} \mathrm{Gr}-1^{-} \mathrm{iNos}^{+}\right.$) in A223 tumors (Fig. 


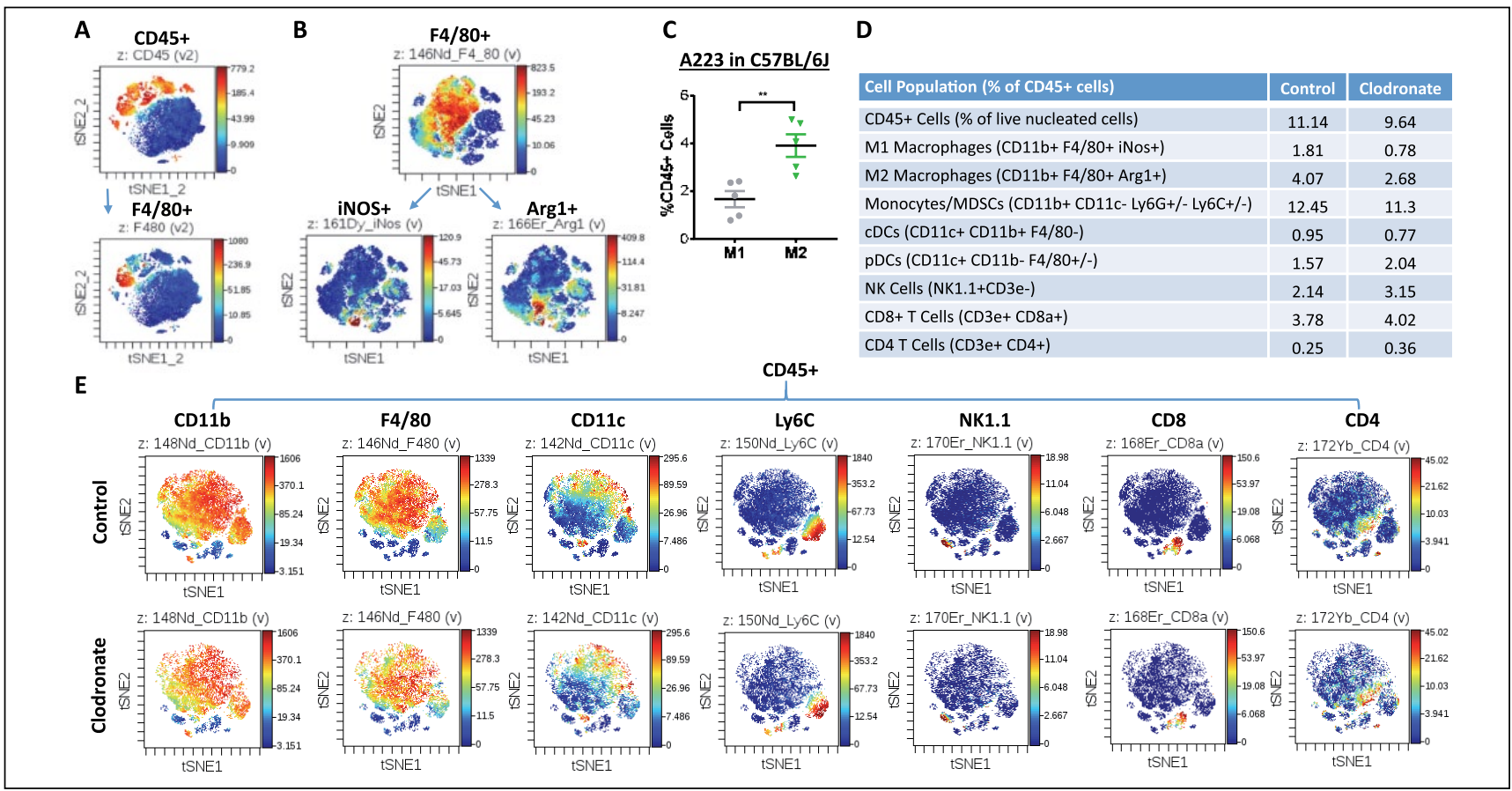

Figure I. Clodronate liposomes' effect on leukocytes in squamous cell carcinoma tumors. Mass cytometry (CyTOF) analyses of leukocytes dervied from A223 tumors grown in C57BL/6] mice. (A) Total leukocytes (marked by CD45; top) and macrophages (marked by F4/80; bottom) are presented in t-SNE maps. F4/80+ macrophages are divided into MI (iNOS+) and M2 (Argl+) subtypes (B) and quantified across 5 tumors (C). Values are presented as mean \pm SEM. ${ }^{* * P}<0.0$ I. Two representative CyTOF analyses of leukocytes in squamous cell carcinomas of control or clodronate-treated mice are quantified $(\mathbf{D})$ and presented in t-SNE maps (E).

1B-D). To deplete macrophages in tumor-bearing mice, we injected clodronate liposomes (van Rooijen and van Nieuwmegen 1984) weekly starting 2 wk after tumor cell injection when tumor volume was $\sim 50 \mathrm{~mm}^{3}$. To assess the relevance of mouse SCCs to human SCCs, we included a human oral SCC line, FaDu, in these experiments. We used CyTOF to screen for changes of tumor-associated leukocytes in C57BL/6 J mice bearing A223 tumors after clodronate treatment (Fig. 1D, E). M1 and M2 macrophages were proportionally reduced by clodronate (Fig. 1D). Additionally, a small number of classic dendritic cells in tumors were slightly reduced (Fig. 1D). We further quantified TAMs as $\mathrm{F} 4 / 80^{+}$cells in tumor sections and verified that clodronate treatment reduced TAMs in all 4 tumor models (Fig. 2A, B). A223 tumors contained vastly more TAMs than B931 tumors or FaDu tumors in athymic recipients, whereas the number of TAMs in A223 tumors did not significantly differ among C57BL/6J recipients or athymic recipients (Fig. 2B), indicating that the number of TAMs is mainly determined by the tumor rather than the host. Systemic macrophage reduction in clodronate-treated mice was confirmed by visibly small spleen sizes and flow cytometry analysis of $\mathrm{F} 4 / 80^{+}$cells in the spleens (Appendix Fig. 1). Because TAMs can suppress $\mathrm{CD}^{+} \mathrm{T}$ cell recruitment to tumors (Peranzoni et al. 2018), we performed flow cytometry to determine if clodronate treatment affected the number of tumorinfiltrating $\mathrm{CD}^{+} \mathrm{T}$ cells. Tumors from clodronate-treated mice had higher numbers of $\mathrm{CD}^{+} \mathrm{T}$ cells (Appendix Fig. 2). To determine if changes in $\mathrm{CD}^{+} \mathrm{T}$ cells have functional impacts on tumor behaviors, we analyzed tumor sizes in clodronatetreated groups in athymic nude mouse recipients (A223, B931, and $\mathrm{FaDu}$ ) and in $\mathrm{C} 57 \mathrm{BL} / 6 \mathrm{~J}$ recipients (A223); all tumors from clodronate-treated groups were significantly smaller than those from control-treated groups (Fig. 2C). Therefore, the effect of TAM reduction on tumor growth was not $\mathrm{T}$ cell dependent. Clodronate treatment did not alter the overall tumor morphology of any tumor line (Appendix Fig. 3). We also compared the B931 tumor metastasis rate in hosts with and without macrophage reduction. Of 24 control mice, 8 developed lung metastasis (33\%); a similar rate was observed in clodronate-treated mice (5 of 21 mice, $23.8 \%, P=0.35$ ). There was no obvious difference in metastasis morphology between the groups (Fig. 2D). E-cadherin, an epithelial cell adhesion molecule lost in B931 tumors, was consistently not restored by macrophage reduction (Fig. 2E). N-cadherin, an adhesion protein aberrantly expressed during epithelial-mesenchymal transition (EMT), was not reduced by macrophage reduction (Fig. 2F).

\section{Macrophage Reduction Had No Effect on SCC Proliferation but Increased SCC Apoptosis}

To determine if reduced tumor growth in clodronate-treated animals was due to reduced tumor cell proliferation, we performed Ki-67 immunofluorescence staining. We did not observe a significant difference in $\mathrm{Ki}-67^{+}$tumor cells between control and clodronate-treated SCCs (Appendix Fig. 4A). 


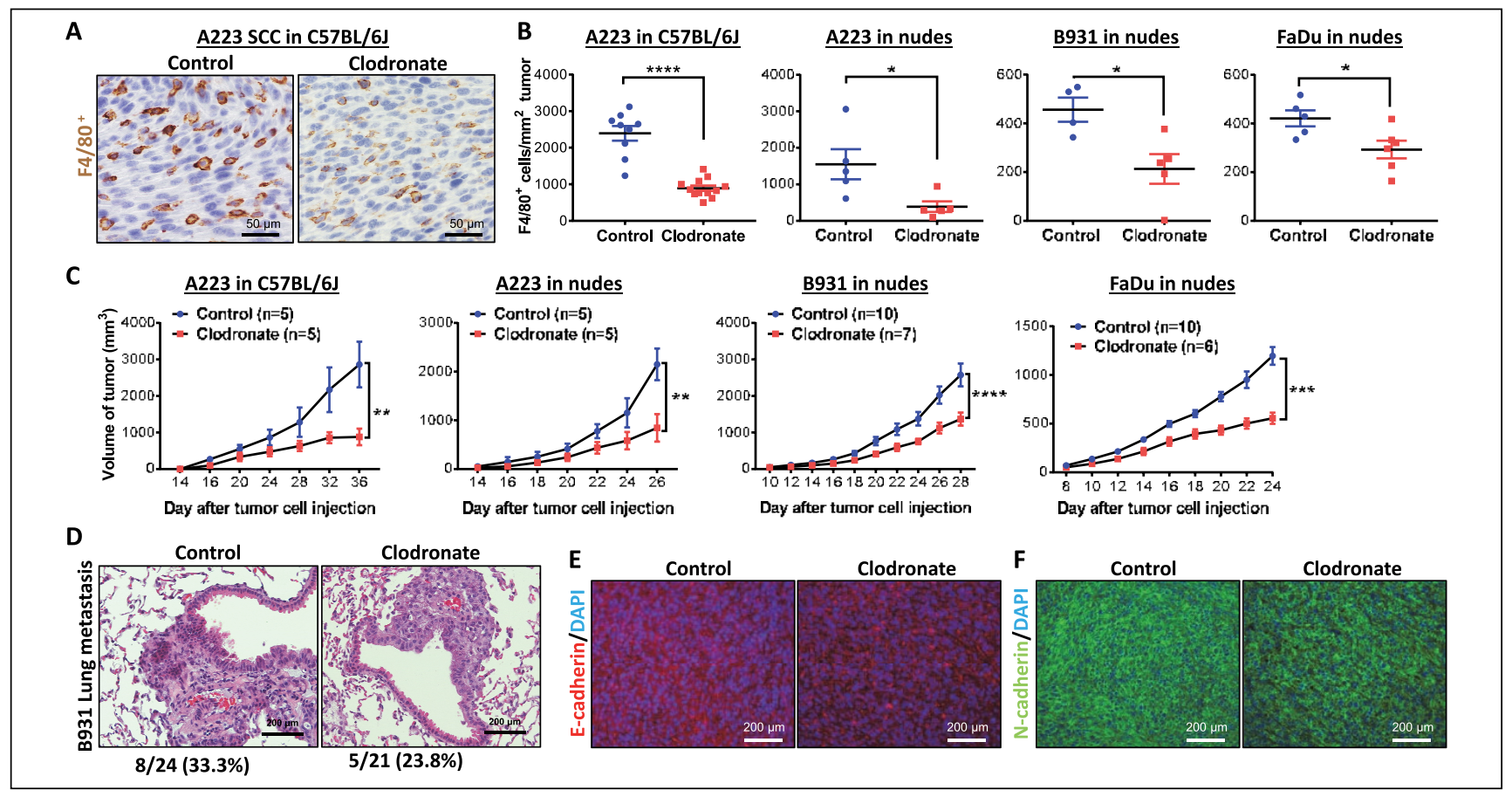

Figure 2. Clodronate-treated mice have blunted squamous cell carcinoma (SCC) tumor volume expansion but not metastasis. (A) Representative

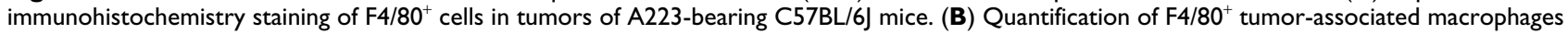
in each tumor type was analyzed by Student's $t$ test. Values are presented as mean \pm SEM. $* P<0.05$, $* * * * P<0.000 I$. (C) Tumors were measured with calipers, and volume was calculated over time; the difference between control and clodronate groups at the final time point was analyzed by Student's $t$ test. Values are presented as mean \pm SEM. $* * P<0.0$ I, $* * * P<0.00$ I, $* * * * P<0.000$ I. (D) Hematoxylin and eosin staining of metastasis-bearing lungs from control and clodronate-treated mice ( $n=24$ for controls and $n=21$ for clodronate-treated mice). Metastatic burden for control and clodronatetreated mice bearing B93I tumors was determined and analyzed by chi-square test $(P=0.35)$. B93। tumors from control and clodronate-treated nude mice were stained for $\mathrm{E}$-cadherin $(\mathbf{E})$ and $\mathrm{N}$-cadherin $(\mathbf{F})$ and counterstained with DAPI.

Similarly, we observed no difference in cell cycle phase (Appendix Fig. 4B, C). Therefore, we next performed TUNEL staining to identify apoptotic cells. Tumors of clodronatetreated mice had more $\mathrm{TUNEL}^{+}$cells than control groups for athymic nude and C57BL/6J recipients (Fig. 3). Consistent with this observation, Western blot analysis revealed that caspase 3 and cleaved caspase 8, mediators of apoptosis (Mantovani et al. 2004; Chanmee et al. 2014), were higher in SCCs with macrophage reduction (Fig. 4A). Conversely, we examined if apoptosis inhibitors were reduced after clodronate treatment. Phosphorylated Bcl2 was barely detectable in most tumor cells in treated and control groups, and Bcl-xL levels, though higher than $\mathrm{pBcl} 2$, were not altered by clodronate treatment (not shown). FLIP, an inhibitor of caspase-8 activation (Mantovani et al. 2004; Chanmee et al. 2014), was lower in clodronate-treated SCCs than control SCCs (Fig. 4A, B). Because FLIP can be produced by either tumor cells or macrophages (Huang et al. 2017; Alkurdi et al. 2018), we performed immunostaining to determine the source of FLIP and found that it was located in tumor epithelial cells and TAMs. In A223 tumors, FLIP $^{+}$cells were uniformly high, and FLIP staining intensity was reduced by clodronate treatment (Fig. 4B). In B931 and FaDu tumors, which have fewer TAMs than A223 tumors, regions with more TAMs also had more LLIP $^{+}$ tumor cells, and clodronate treatment reduced the number and staining intensity of $\mathrm{FLIP}^{+}$tumor cells (Fig. 4B). Because macrophage-secreted TNF $\alpha$ can induce tumor cell apoptosis and paradoxically activate prosurvival NFkB signaling (Urban et al. 1986; Duffey et al. 2000; You et al. 2001), we examined $\mathrm{TNF} \alpha$ levels in sera of tumor-bearing mice and nuclear NFKB p-p65 in tumors, a surrogate marker for NFKB activation. $\mathrm{TNF} \alpha$ serum level was very low in control and clodronatetreated tumor-bearing mice (not shown). Nuclear NFkB p-p65 was seen in most tumor cells in control groups but was reduced in clodronate-treated tumors (Fig. 4C). To determine if endogenous NFKB activity in tumor cells contributed to FLIP expression, we treated mouse and human SCC cells with an IkB kinase (IKK) inhibitor, BMS345541, and found that FLIP expression was reduced with the IkB kinase (IKK) inhibitor but not by TGF $\beta$ inhibitor LY2109761 (Fig. 4D). However, application of exogenous TNF $\alpha$ was insufficient to increase FLIP expression (Fig. 4E), suggesting that $\mathrm{NFKB}$ regulation of FLIP is independent of TNF $\alpha$ in our SCC models.

\section{Macrophage Ablation Decreased Vascular Density in SCCs}

We further evaluated the pathology of SCCs with and without clodronate treatment and identified that vessel density was reduced in SCCs from mice treated with clodronate 

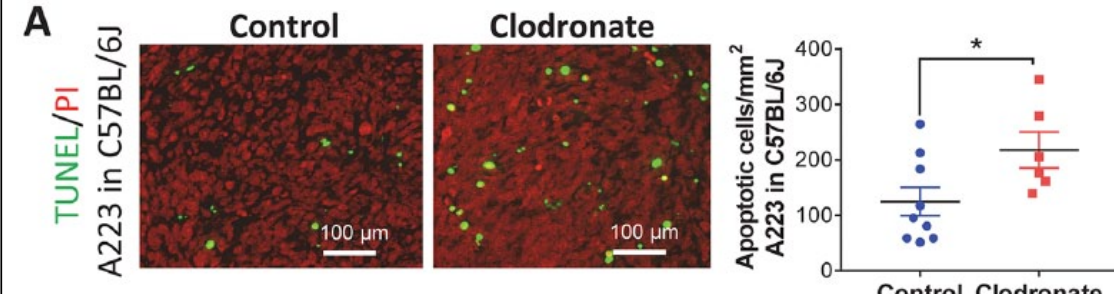

B
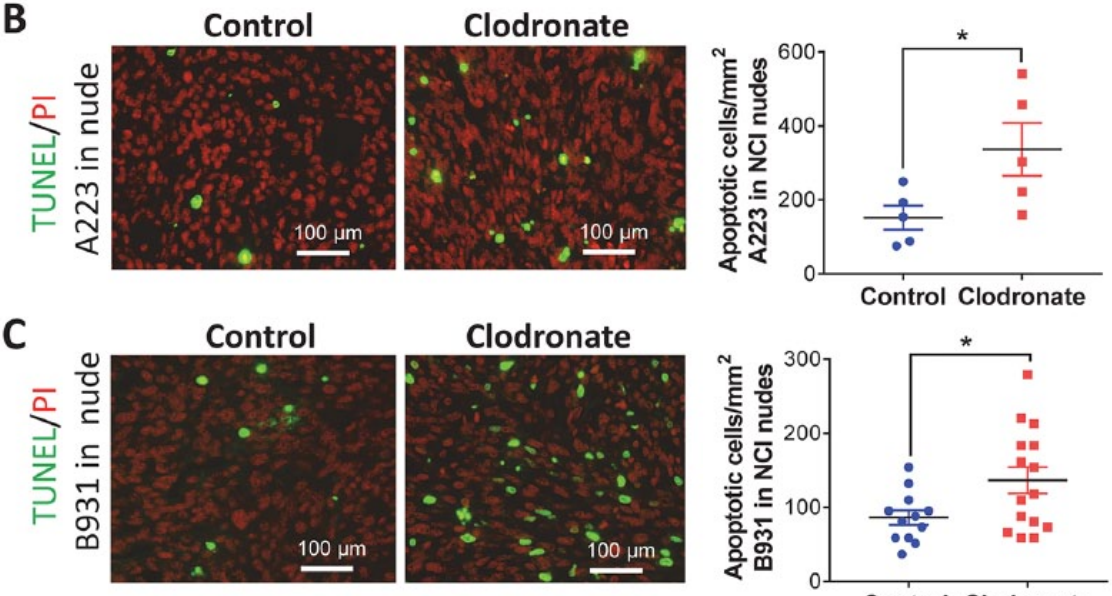

Clodronate

D

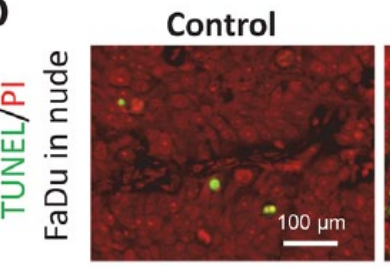

Clodronate
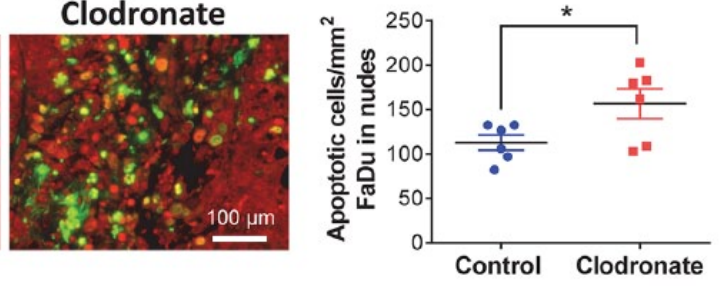

Figure 3. Increased apoptosis in squamous cell carcinomas (SCCs) from clodronate-treated mice. Apoptotic cells were identified by TUNEL staining and nuclei by propidium iodide (PI) staining, quantified in each SCC model, and analyzed by Student's $t$ test. (A) A223 tumors grown in C57BL/6] mice. (B) A223 tumors grown in athymic nude mice. (C) B93I tumors grown in athymic nude mice. (D) FaDu tumors grown in athymic nude mice. Values are presented as mean $\pm \mathrm{SEM}$. $* P<0.05$.

(CD31) in the vascular area with or without macrophage reduction (Appendix Fig. 5), suggesting that reduced vascular density is primarily associated with VEGFA-mediated angiogenesis.

\section{Discussion}

Although roles of TAMs in cancer development and progression have been studied extensively, the functional impact of TAMs varies among tumor types and stages and is less well known. We show that in SCCs, TAM ablation reduced tumor volumes primarily as a result of increased apoptosis and reduced angiogenesis.

It was a bit surprising that TAM reduction did not affect tumor cell proliferation, given TAM's wellknown role in producing growth factors and cytokines promoting cancer cell proliferation (Mantovani et al. 2017). Notably, clodronate-mediated TAM depletion was incomplete, and the depletion was not as robust as the macrophage depletion in the spleen (Figs. 1, 2 vs. Appendix Fig. 1). Therefore, it is possible that a more profound TAM depletion is needed to reduce tumor cell proliferation; conversely, the tumor microenvironment could help TAMs survive clondronate treatment better than macrophages outside of tumors. The lack of reduced proliferation by clondronate in these

(Fig. 5A, B). Vascular endothelial growth factor A (VEGFA), a prominent mediator of angiogenesis (Leung et al. 1989), was reduced in clodronate-treated tumors (Fig. 5C). VEGFA was found in tumors cells and TAMs. VEGFA levels were reduced in tumor cells of clodronate-treated mice (Fig. 5D), suggesting that macrophages secrete or govern a systemic factor that regulates VEGFA. We therefore examined TGF $\beta 1$, a known VEGFA regulator (Clifford et al. 2008). ELISA revealed reduced TGF $\beta 1$ in sera of clodronate-treated tumor-bearing mice (Fig. 5E). Phosphorylated Smad3 (pSmad3), a surrogate marker of TGF $\beta$ activation, was consistently reduced in clodronate-treated SCCs (Fig. 5F, G). Furthermore, although F4/80 ${ }^{+}$TAMs were positive for pSmad3, pSmad3 was mainly reduced in tumor epithelial cells in clodronate-treated mice (Fig. 5G). Because TGF $\beta$ can also mediate angiogenesis through pSmad1/5/8 (Goumans et al. 2003), we performed double immunofluorescence staining of CD31 and pSmad1/5/8. We found no difference in pSmad1/5/8-positive endothelium
SCCs could also be due to their escape from regulatory mechanisms of cell cycle arrest. Since mouse and human SCCs in this study harbored homozygous Smad4 loss and oncogenic Ras activation, these tumors escaped from TGF $\beta$-mediated growth arrest due to loss of Smad4, the critical mediator of this process. Another possibility is that these SCCs have an autonomous growth signal from activated Ras or produce sufficient growth factors to sustain their growth in the absence of macrophages. In contrast, we found increased apoptosis after macrophage ablation that appeared to contribute to reduced tumor volumes in these SCCs. The fact that increased apoptosis occurred in immune-compromised (athymic) and immune-competent $(\mathrm{C} 57 \mathrm{BL} / 6 \mathrm{~J})$ conditions suggests that apoptosis is a $\mathrm{T}$ cell-independent effect of macrophage depletion and that T cell-mediated antitumor immunity is not required. Although higher levels of $\mathrm{CD}^{+} \mathrm{T}$ cells in tumors of clodronate-treated mice were observed, our previous study showed that $\mathrm{CD}^{+} \mathrm{T}$ cells in this tumor model express PD1 and LAG3 inhibitory receptors 
A

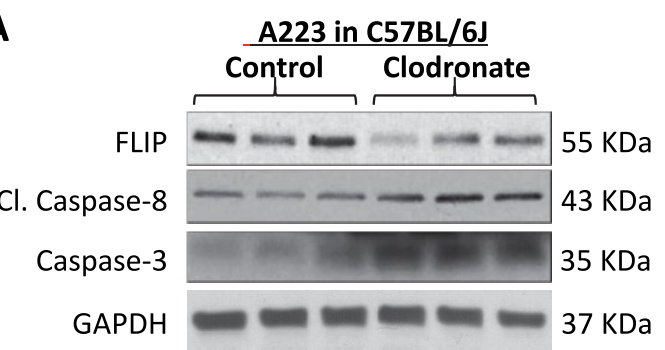

B

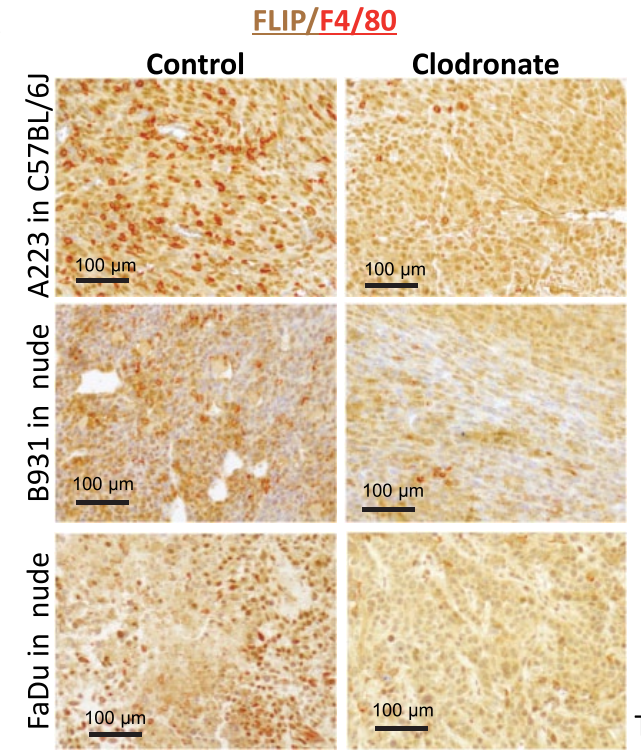

C

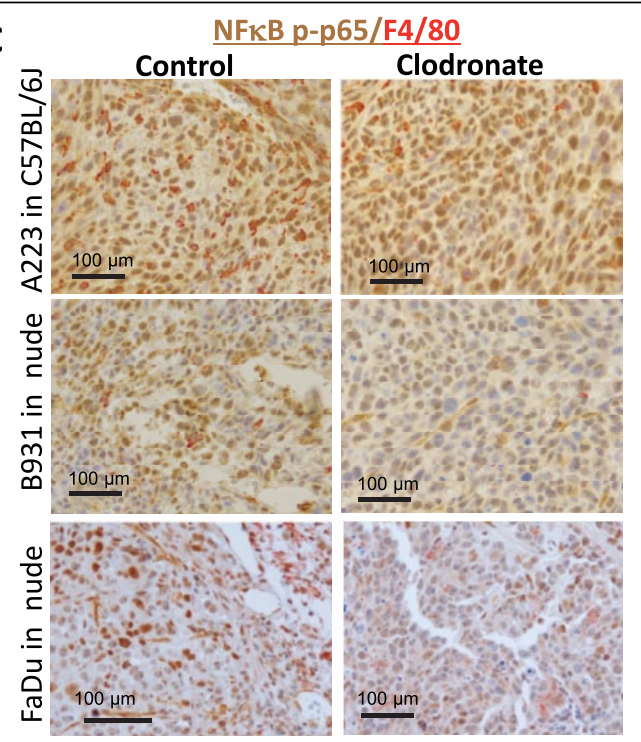

D
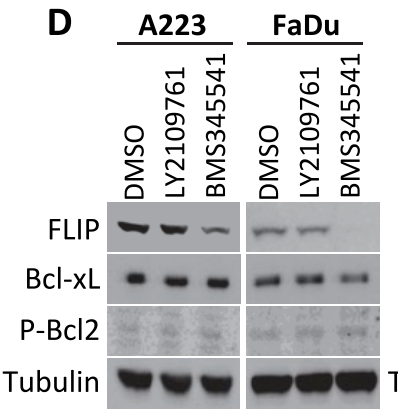

E

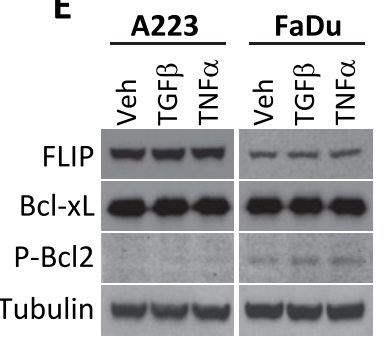

Figure 4. Clodronate-mediated apoptosis correlates with decreased NFKB-dependent FLIP expression. (A) A223 tumors from control and clodronate-treated C57BL/6] mice were lysed and evaluated by Western blot with the indicated antibodies. Immunohistochemistry staining of FLIP and F4/80 (B) or NFKB P-p65 and F4/80 (C) in 3 squamous cell carcinoma tumor models. (D, E) A223 or FaDu cells were treated with DMSO, TGF $\beta R$ inhibitor (LY2I 0976I), IKK inhibitor (BKM34554I), recombinant TGF $\beta$, or recombinant TNF $\alpha$ as described in the Methods and Materials. Western blot analysis was performed with the indicated antibodies.

(Mishra et al. 2016). Therefore, increased CD $8^{+} \mathrm{T}$ cells may not contain sufficient numbers of activated $\mathrm{CD} 8^{+} \mathrm{T}$ cells to further reduce tumor growth.

Among apoptosis regulators, FLIP, an apoptosis inhibitor, can be produced by tumor cells and TAMs (Huang et al. 2010; Zhao et al. 2011). Therefore, the reduced FLIP in tumor cells of clodronate-treated mice suggests that FLIP functionally contributes to tumor cell protection. Our study reveals that FLIP is primarily produced by tumor epithelial cells in areas with high numbers of TAMs. These data suggest that FLIP expression in tumor epithelial cells is likely a result of TAMtumor cell interactions not necessarily restricted by cell-cell contact and that macrophage depletion contributed to epithelial FLIP reduction. It has been shown that $\mathrm{NF \kappa B}$ activation in cancer cells attenuates TNF $\alpha$-mediated killing of tumor cells (Urban et al. 1986; You et al. 2001; Humphreys et al. 2018). Tumors in this study had little TNF $\alpha$ and fewer M1 than M2 TAMs, suggesting that TAM-induced tumor cell killing is largely absent in these tumors. Our data suggest that constitutive NF $\kappa$ B activation in these tumor cells could be one of the major pathways to induce FLIP expression. This notion is further supported by the data that $\mathrm{NF} \kappa \mathrm{B}$ inhibitor reduced FLIP protein in vitro and reduced FLIP levels in tumor cells correlated with reduced $\mathrm{NF} \kappa \mathrm{B}$ activation in vivo after clondronate treatment. Furthermore, because TNF $\alpha$ did not induce FLIP protein expression, our data suggest that TNF $\alpha$ independent NF $\kappa \mathrm{B}$ activation largely contributes to FLIP production. To this end, TGF $\beta$-mediated TNF $\alpha$-independent NF $\kappa$ B activation has been shown to be tumor promotive (Freudlsperger et al. 2013). Therefore, reduced TGF $\beta$ signaling by clodronate treatment could also indirectly contribute to reduced FLIP production. However, because TGF $\beta$ inhibitor did not reduce FLIP levels, our data suggest that FLIP production is primarily regulated by $\mathrm{NF} \kappa \mathrm{B}$ activation. A primary mechanism of FLIP activity is to inhibit activation of extrinsic cell death signals that activate caspase 8 or caspase 10 activity (Huang et al. 2010; Zhao et al. 2011; Humphreys et al. 2018). Thus, the increased levels of cleaved (activated) caspase 8 observed in tumors of clodronate-treated mice are consistent with a dearth of FLIP-mediated inhibition of extrinsic death cues regulated via caspase 8 activation. Additionally, reduced angiogenesis by TAM ablation could indirectly result in 


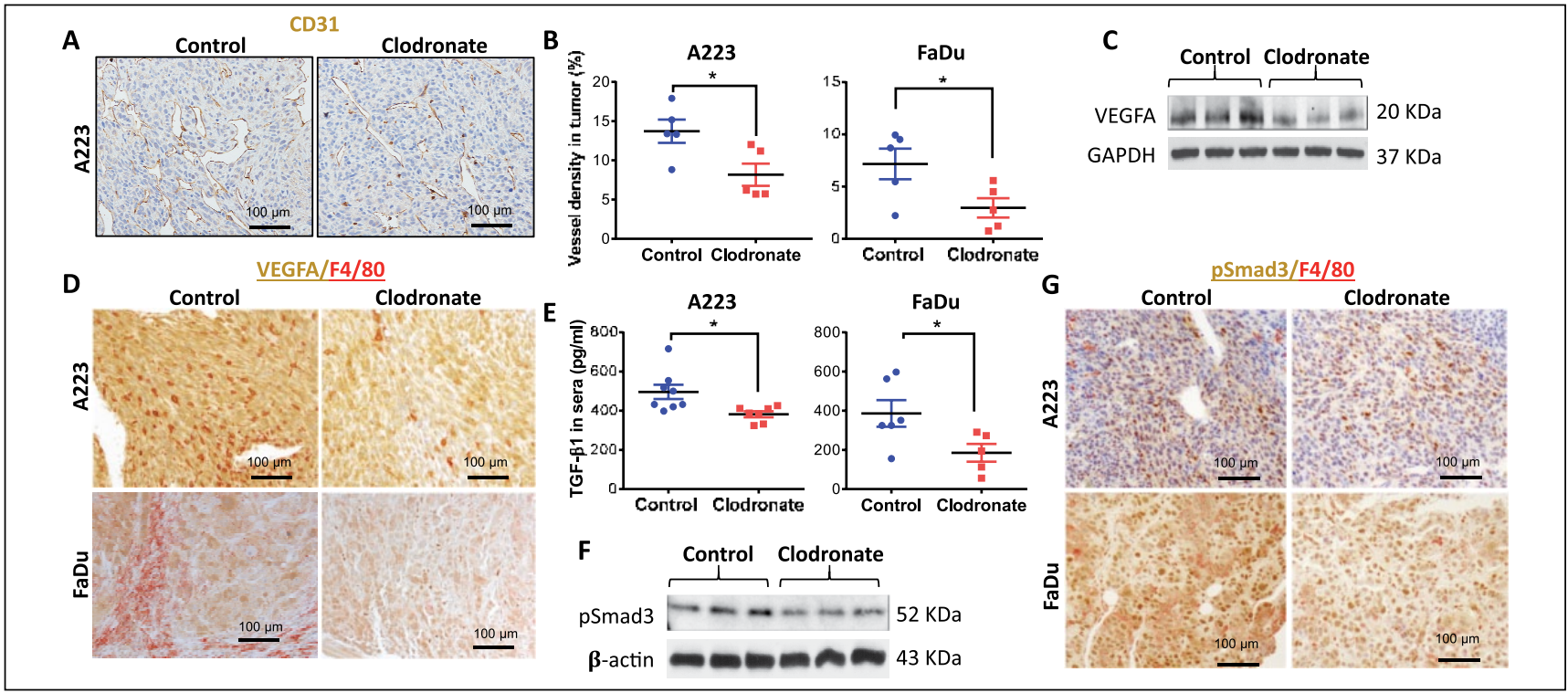

Figure 5. Macrophage-depleted tumors have decreased blood vessel density and angiogenic factors. (A) Blood vessels in A223 tumors in C57BL/6 mice were identified by immunohistochemistry staining for endothelium marker CD3I. (B) Vessel lumen size was quantified as a percentage of tumor area for A223 and FaDu tumors. (C) Levels of VEGFA and GAPDH in A223 tumor lysates were determined by Western blot analysis. (D) Immunohistochemistry staining of VEGFA and F4/80 in A223 tumors grown in C57BL/6) mice or FaDu tumors grown in athymic nude mice. (E) An ELISA kit was used to detect TGF $\beta$ I levels in the sera of $A 223$ tumor-bearing C57BL/6J mice or FaDu tumor-bearing athymic nude mice treated with control or clodronate liposomes. (F) Levels of pSmad3 and $\beta$-actin in A223 tumor lysates were determined by Western blot analysis. (G) Immunohistochemistry staining of pSmad3 and F4/80 in A223 tumors grown in C57BL/6] mice and FaDu tumors grown in athymic nude mice. Statistics by Student's $t$ test; $* P<0.05$. (B, E) Values are presented as mean \pm SEM.

increased apoptosis from a shortage of nutrients and oxygen delivered to tumor cells.

TAMs are well-known regulators of tumor angiogenesis (Liss et al. 2001; De Palma et al. 2017); thus, it is not surprising that clondronate-treated SCCs had reduced vessel densities in our study. We found that VEGFA reduction was primarily located in tumor epithelial cells. This could be a consequence of systemic changes to cytokines as a result of macrophage depletion. For example, TGF $\beta 1$ was reduced in serum from macrophage-depleted SCC-bearing mice. Because VEGFA is a major TGF $\beta$ transcriptional target that is dependent on Smad2/3/4 (Clifford et al. 2008), circulating TGF $\beta 1$ could drive VEGFA production in tumor epithelial cells. Conversely, decreased TGF $\beta 1$ in clodronate-treated mice would be expected to induce less VEGFA expression in tumor cells. Decreased tumor cell TGF $\beta$ signaling in clodronate-treated mice is supported by the observation that these mice have reduced levels of tumor pSmad3. With reduced TGF $\beta 1$ and pSmad3, VEGFA production in tumor cells could also be reduced, especially in the absence of Smad4. Therefore, it is possible that even if TGF $\beta 1$ reduction is insufficient to reduce endothelial Smad1/5/8-dependent angiogenesis, it could be sufficient to reduce VEGFA-dependent angiogenesis after TAM ablation.

Lowered pSmad 3 in tumor epithelial cells suggests that reduced TGF $\beta 1$ could primarily affect tumor epithelia. However, this reduction appears to be insufficient to reverse EMT, which correlated with the lack of metastasis reduction in clondronate-treated tumors. This result suggests that either the TGF $\beta 1$ level is not low enough to attenuate EMT or metastasis with macrophage ablation or, more likely, other EMT regulators are the primary regulators given that these SCCs do not have Smad4 to mediate TGF $\beta 1$-induced EMT. In summary, our study identified an overall protumor role for macrophages in SCCs, independent of T lymphocytes. Ablation of macrophages led to increased apoptosis and decreased angiogenesis in the tumor microenvironment. Intriguingly, macrophage depletion did not influence the rate of metastasis. Future studies will determine if findings in this study apply more broadly to human SCCs beyond SMAD4 loss and Ras activation. If this is the case, targeting TAM-mediated tumor expansion could extend the treatment window for other therapeutic interventions.

\section{Author Contributions}

F.L. Wu, contributed to conception, design, data acquisition, analysis, and interpretation, drafted and critically revised the manuscript; K. Nolan, L. Bian, contributed to data acquisition, analysis, and interpretation, critically revised the manuscript; A.A. Strait, J.H. Wang, A. Jimeno, contributed to data analysis and interpretation, critically revised the manuscript; K.A. Nguyen, contributed to data acquisition, critically revised the manuscript; H.M. Zhou, contributed to design, data analysis, and interpretation, critically revised the manuscript; C.D. Young, contributed to design, data acquisition, analysis, and interpretation, drafted and critically revised the manuscript; X.J. Wang, contributed to conception, design, data analysis, and interpretation, drafted and critically 
revised the manuscript. All authors gave final approval and agree to be accountable for all aspects of the work.

\section{Acknowledgments}

This work was supported by VA Merit Award I01BX003232 to X.J.W., National Institutes of Health grants R01DE24371 to A.J. and X.J.W. and R01DE027329 to J.H.W. and X.J.W., the National Nature Science Foundation of China No. 81772898 to H.M.Z., and China Scholarship Council No. 201606240201 to F.L.W. Flow cytometry experiments were performed at the University of Colorado Flow Cytometry Shared Resource, funded in part by a National Cancer Institute Cancer Center Support Grant (P30CA046934). The authors declare no potential conflicts of interest with respect to the authorship and/or publication of this article.

\section{References}

Alkurdi L, Virard F, Vanbervliet B, Weber K, Toscano F, Bonnin M, Le Stang N, Lantuejoul S, Micheau O, Renno T, et al. 2018. Release of c-FLIP brake selectively sensitizes human cancer cells to TLR3-mediated apoptosis. Cell Death Dis. 9(9):874.

Barretina J, Caponigro G, Stransky N, Venkatesan K, Margolin AA, Kim S, Wilson CJ, Lehar J, Kryukov GV, Sonkin D, et al. 2012. The cancer cell line encyclopedia enables predictive modelling of anticancer drug sensitivity. Nature. 483(7391):603-607.

Biswas SK, Mantovani A. 2010. Macrophage plasticity and interaction with lymphocyte subsets: cancer as a paradigm. Nat Immunol. 11(10):889-896.

Chanmee T, Ontong P, Konno K, Itano N. 2014. Tumor-associated macrophages as major players in the tumor microenvironment. Cancers (Basel). 6(3):1670-1690.

Clifford RL, Deacon K, Knox AJ. 2008. Novel regulation of vascular endothelial growth factor-A (VEGF-A) by transforming growth factor (beta)1: requirement for Smads, (beta)-catenin, and GSK3(beta). J Biol Chem. 283(51):35337-35353

De Palma M, Biziato D, Petrova TV. 2017. Microenvironmental regulation of tumour angiogenesis. Nate Rev Cancer. 17(8):457-474.

Duffey DC, Crowl-Bancroft CV, Chen Z, Ondrey FG, Nejad-Sattari M, Dong G, Van Waes C. 2000. Inhibition of transcription factor nuclear factorkappaB by a mutant inhibitor-kappaBalpha attenuates resistance of human head and neck squamous cell carcinoma to TNF-alpha caspase-mediated cell death.. Br J Cancer. 83(10):1367-1374.

Freudlsperger C, Bian Y, Contag Wise S, Burnett J, Coupar J, Yang X, Chen Z, Van Waes C. 2013. TGF-beta and NF-kappab signal pathway cross-talk is mediated through TAK1 and SMAD7 in a subset of head and neck cancers. Oncogene. 32(12):1549-1559.

Goumans MJ, Valdimarsdottir G, Itoh S, Lebrin F, Larsson J, Mummery C, Karlsson S, ten Dijke P. 2003. Activin receptor-like kinase (ALK)1 is an antagonistic mediator of lateral TGFbeta/ALK5 signaling. Mol Cell. 12(4):817-828.

Hernandez AL, Wang Y, Somerset HL, Keysar SB, Aisner DL, Marshall C, Bowles DW, Karam SD, Raben D, Jimeno A, et al. 2018. Inter- and intratumor heterogeneity of SMAD4 loss in head and neck squamous cell carcinomas. Mol Carcinog. 58(5):666-673.

Huang QQ, Birkett R, Doyle RE, Haines GK, Perlman H, Shi B, Homan P, Xing L, Pope RM. 2017. Association of increased F4/80(high) macrophages with suppression of serum-transfer arthritis in mice with reduced flip in myeloid cells. Arthritis Rheumatol. 69(9):1762-1771.

Huang QQ, Perlman H, Huang Z, Birkett R, Kan L, Agrawal H, Misharin A, Gurbuxani S, Crispino JD, Pope RM. 2010. Flip: a novel regulator of macrophage differentiation and granulocyte homeostasis. Blood. 116(23):4968-4977.

Humphreys L, Espona-Fiedler M, Longley DB. 2018. Flip as a therapeutic target in cancer. FEBS J. 285(22):4104-4123.

Jih DM, Lyle S, Elenitsas R, Elder DE, Cotsarelis G. 1999. Cytokeratin 15 expression in trichoepitheliomas and a subset of basal cell carcinomas sug- gests they originate from hair follicle stem cells. J Cutan Pathol. 26(3):113118.

Leung DW, Cachianes G, Kuang WJ, Goeddel DV, Ferrara N. 1989. Vascular endothelial growth factor is a secreted angiogenic mitogen. Science. 246(4935):1306-1309.

Liss C, Fekete MJ, Hasina R, Lam CD, Lingen MW. 2001. Paracrine angiogenic loop between head-and-neck squamous-cell carcinomas and macrophages. Int J Cancer. 93(6):781-785.

Mantovani A, Marchesi F, Malesci A, Laghi L, Allavena P. 2017. Tumourassociated macrophages as treatment targets in oncology. Nat Rev Clin Oncol. 14(7):399-416.

Mantovani A, Sica A, Sozzani S, Allavena P, Vecchi A, Locati M. 2004. The chemokine system in diverse forms of macrophage activation and polarization. Trends Immunol. 25(12):677-686.

Mishra AK, Kadoishi T, Wang X, Driver E, Chen Z, Wang XJ, Wang JH. 2016. Squamous cell carcinomas escape immune surveillance via inducing chronic activation and exhaustion of CD8+ T Cells co-expressing PD-1 and LAG-3 inhibitory receptors. Oncotarget. 7(49):81341-81356.

Murray PJ. 2017. Macrophage polarization. Annu Rev Physiol. 79:w541-w566.

Noy R, Pollard JW. 2014. Tumor-associated macrophages: from mechanisms to therapy. Immunity. 41(1):49-61.

Okubo M, Kioi M, Nakashima H, Sugiura K, Mitsudo K, Aoki I, Taniguchi H, Tohnai I. 2016. M2-polarized macrophages contribute to neovasculogenesis, leading to relapse of oral cancer following radiation. Sci Rep. 6:27548.

Peranzoni E, Lemoine J, Vimeux L, Feuillet V, Barrin S, Kantari-Mimoun C, Bercovici N, Guerin M, Biton J, Ouakrim H, et al. 2018. Macrophages impede CD8 $t$ cells from reaching tumor cells and limit the efficacy of antiPD-1 treatment. Proc Natl Acad Sci U S A. 115(17):E4041-E4050.

Pirila E, Vayrynen O, Sundquist E, Pakkila K, Nyberg P, Nurmenniemi S, Paakkonen V, Pesonen P, Dayan D, Vered M, et al. 2015. Macrophages modulate migration and invasion of human tongue squamous cell carcinoma. PLoS One. 10(3):e0120895.

Qian BZ, Pollard JW. 2010. Macrophage diversity enhances tumor progression and metastasis. Cell. 141(1):39-51.

Ruffell B, Coussens LM. 2015. Macrophages and therapeutic resistance in cancer. Cancer Cell. 27(4):462-472.

Saranath D, Chang SE, Bhoite LT, Panchal RG, Kerr IB, Mehta AR, Johnson NW, Deo MG. 1991. High frequency mutation in codons 12 and 61 of $\mathrm{H}$-ras oncogene in chewing tobacco-related human oral carcinoma in India. Br J Cancer. 63(4):573-578.

Tudor D, Locke M, Owen-Jones E, Mackenzie IC. 2004. Intrinsic patterns of behavior of epithelial stem cells. J Investig Dermatol Symp Proc. 9(3):208214.

Urban JL, Shepard HM, Rothstein JL, Sugarman BJ, Schreiber H. 1986. Tumor necrosis factor: a potent effector molecule for tumor cell killing by activated macrophages. Proc Natl Acad Sci U S A. 83(14):5233-5237.

van Rooijen N, van Nieuwmegen R. 1984. Elimination of phagocytic cells in the spleen after intravenous injection of liposome-encapsulated dichloromethylene diphosphonate: an enzyme-histochemical study. Cell Tissue Res. 238(2):355-358.

Weber M, Iliopoulos C, Moebius P, Buttner-Herold M, Amann K, Ries J, Preidl R, Neukam FW, Wehrhan F. 2016. Prognostic significance of macrophage polarization in early stage oral squamous cell carcinomas. Oral Oncol. 52:75-84.

White RA, Neiman JM, Reddi A, Han G, Birlea S, Mitra D, Dionne L, Fernandez P, Murao K, Bian L, et al. 2013. Epithelial stem cell mutations that promote squamous cell carcinoma metastasis. J Clin Invest. 123(10):4390-4404.

You Z, Ouyang H, Lopatin D, Polver PJ, Wang CY. 2001. Nuclear factor-kappa $\mathrm{B}$-inducible death effector domain-containing protein suppresses tumor necrosis factor-mediated apoptosis by inhibiting caspase-8 activity. J Biol Chem. 276(28):26398-26404.

Yuan A, Hsiao YJ, Chen HY, Chen HW, Ho CC, Chen YY, Liu YC, Hong TH, Yu SL, Chen JJ, et al. 2015. Opposite effects of M1 and M2 macrophage subtypes on lung cancer progression. Sci Rep. 5:14273.

Zhang M, He Y, Sun X, Li Q, Wang W, Zhao A, Di W. 2014. A high M1/M2 ratio of tumor-associated macrophages is associated with extended survival in ovarian cancer patients. J Ovarian Res. 7:19.

Zhao L, Yue P, Lonial S, Khuri FR, Sun SY. 2011. The NEDD8-activating enzyme inhibitor, MLN4924, cooperates with TRAIL to augment apoptosis through facilitating c-FLIP degradation in head and neck cancer cells. Mol Cancer Ther. 10(12):2415-2425. 